\title{
Role of the CacyBP/SIP protein in gastric cancer
}

\author{
HUIHONG ZHAI ${ }^{1}$, JUAN MENG ${ }^{1}$, HAIFENG JIN ${ }^{2}$, YUANFEI $\mathrm{LI}^{3}$ and JINBO WANG ${ }^{2}$ \\ ${ }^{1}$ Department of Digestive Diseases, General Hospital of Ningxia Medical University, Yinchuan, \\ Ningxia Hui Autonomous Region 750004; ${ }^{2}$ State Key Laboratory of Cancer Biology, Institute of Digestive Diseases and \\ ${ }^{3}$ Department of Pathology, Xijing Hospital, Fourth Military Medical University, Xi'an, Shaanxi 710032, P.R. China
}

Received March 13, 2014; Accepted October 22, 2014

DOI: $10.3892 / \mathrm{ol} .2015 .3059$

\begin{abstract}
Various reports indicate that calcyclin binding protein/Siah-1-interacting protein (CacyBP/SIP) is an important protein in tumorigenesis, but whether CacyBP/SIP promotes or suppresses cancer may depend on the cell type. In order to investigate whether CacyBP/SIP is significant in gastric cancerous tumorigenesis, the present study used immunohistochemistry to analyze 181 gastric cancer tissue samples, as well as 181 healthy tissue samples from the same gastric cancer patients. The immunohistochemical results were compared against patient data and pathological analysis of the tissue slices, including gender, age, degree of tumor differentiation and tumor, node, metastasis (TNM) stage. In addition, the level of CacyBP/SIP expression was detected in three frozen tissue samples of gastric adenocarcinoma using western blot analysis. Of the 181 cases analyzed in the present study, 80 cases were identified as non-metastatic gastric cancer and 101 cases were identified as gastric cancer that had metastasized to the lymph nodes. Tissue biopsies from the two sets of patients were examined using immunohistochemistry to identify the level of CacyBP/SIP expression in metastatic and primary gastric cancer tissues. Statistical analyses were performed on all data. The immunohistochemical analysis revealed that CacyBP/SIP was expressed in $31 \%(56 / 181)$ of gastric adenocarcinoma tissue samples and 7\% (12/181) of adjacent non-cancerous gastric tissues $(\mathrm{P}<0.05)$. Furthermore, the expression levels of CacyBP/SIP were higher in cancerous tissue compared with the adjacent non-cancerous gastric tissue using western blotting. No association was identified between CacyBP/SIP expression and patient age $(\mathrm{P}=0.975)$, gender $(\mathrm{P}=0.185)$, degree of tumor differentiation $(\mathrm{P}=0.076)$ or TNM stage $(\mathrm{P}=0.979)$. Among the 101 patients with metastatic
\end{abstract}

Correspondence to: Professor Huihong Zhai, Department of Digestive Diseases, General Hospital of Ningxia Medical University, 804 Shengli Street, Yinchuan, Ningxia Hui Autonomous Region 750004, P.R. China

E-mail: zhaihuihong@263.net

Abbreviation: CacyBP/SIP, calcyclin binding protein/Siah-1 interacting protein

Key words: CacyBP/SIP, gastric cancer, immunohistochemistry, metastasis gastric cancer, CacyBP/SIP was expressed at primary sites in $31 \%(31 / 101)$ of cases and at metastatic sites in $26 \%(26 / 101)$ of cases $(\mathrm{P}=0.434)$. However, among the 80 patients with non-metastatic gastric cancer, CacyBP/SIP was expressed at the tumor site in $34 \%(27 / 80)$ of cases, which was not significantly different from the $31 \%(25 / 80)$ of cases in the metastatic group $(\mathrm{P}=0.662)$. These findings indicate that $\mathrm{CacyBP} / \mathrm{SIP}$ expression is not a marker of gastric cancer or metastatic gastric cancer, nor does it appear to correlate with the clinicopathological features of gastric cancer.

\section{Introduction}

Calcyclin binding protein (CacyBP) was initially described as a binding partner of calcylin (S100A6) at physiological $\mathrm{Ca}^{2+}$ concentrations in Ehrlich ascites tumor cells (1). Three years later, Matsuzawa and Reed (2) identified that the human analog of mouse CacyBP interacts with Siah-1, and this protein was termed Siah-1-interacting protein (SIP). Thus, the protein is now referred to as CacyBP/SIP.

Various reports propose a role for CacyBP/SIP in cellular processes, such as ubiquitination (2), proliferation (3), differentiation $(4,5)$, tumorigenesis, cytoskeletal rearrangement $(6,7)$ and transcriptional regulation (8). In our previous study, it was identified that CacyBP is expressed at higher levels in multidrug-resistant gastric cancer cells (SGC7901/adriamycin) compared with the parental cell line (SGC7901) (9). Thus, upregulation of CacyBP/SIP may enhance the resistance of gastric cancer cells to various types of chemotherapeutic agents, whereas downregulation of CacyBP/SIP may partially reverse the drug-resistant properties of gastric cancer cells (10). Thus, to understand the function of CacyBP in cancer, our previous study produced three monoclonal antibodies against CacyBP (11). Using these antibodies, it was identified that numerous types of healthy tissue, including gastric tissue, exhibited minimal expression of CacyBP/SIP, whereas numerous types of cancerous tissue expressed, or even overexpressed, CacyBP/SIP (12).

Additional studies have implicated CacyBP/SIP in tumorigenesis, although whether it promotes or suppresses cancer appears to depend on the cell type. For example, overexpressed CacyBP/SIP suppressed the growth of renal and gastric cancer cells $(13,14)$. However, CacyBP/SIP expression levels were significantly increased in pancreatic cancer tissue compared with adjacent non-cancerous pancreatic tissue, and 
the expression level was associated with the degree of tumor differentiation, higher tumor, node, metastasis (TNM) stage and distal metastasis (15). Additionally, CacyBP/SIP expression levels were higher in more clinically advanced breast cancer, including metastatic breast cancer (16).

Considering the opposing roles of CacyBP/SIP in cancer tumorigenesis, the present study aimed to determine the whether CacyBP acts as a suppressor or promoter of tumorigenesis in gastric cancer. Thus, immunohistochemical analysis of tissue samples from gastric cancer patients was performed to detect the expression levels of CacyBP/SIP, and to compare CacyBP/SIP expression levels between metastatic and non-metastatic cancer tissue.

\section{Materials and methods}

Patients and tissue specimens. Formalin-fixed, paraffin-embedded tumor tissues were obtained from the General Hospital of Ningxia Medical University (Yinchuan, China). A total of 181 samples of gastric adenocarcinoma tissue and adjacent non-cancerous gastric tissues were collected from the same patients. The patients included 80 cases of non-metastatic gastric cancer and 101 cases of gastric cancer that had metastasized to the lymph nodes. None of the patients had undergone preoperative radiotherapy or chemotherapy. In addition, three frozen tissue samples of gastric adenocarcinoma were used in the present study, which were obtained from the General Hospital of Ningxia Medical University. The diagnoses of the paraffin-embedded and fresh tissue specimens were independently established by two experienced pathologists, according to the National Comprehensive Cancer Network guidelines for gastric cancer (2013) (17). This study was approved by the ethics committee of the General Hospital of Ningxia Medical University and written informed consent was obtained from all patients.

Immunohistochemical staining and evaluation. CacyBP/SIP expression levels were detected in the 5- $\mu$ mparaffin-embedded tissue sections using PV-6002 Power Vision Two-Step Histostaining Reagent, according to the manufacturer's instructions (Dako North America, Inc., Carpinteria, CA, USA). The antibody used for immunohistochemistry was a CacyBP/SIP-specific mouse anti-human monoclonal antibody prepared in our laboratory as previously described (clone EA1; dilution, 1:150; initial concentration, $2.1 \mathrm{mg} / \mathrm{ml}$ ) (11). Negative controls were prepared by replacing the primary antibody with pre-immune mouse serum (Beyotime Institute of Biotechnology, Beijing, China). The immunohistochemical stains were independently evaluated by two pathologists who were not aware of the status of the samples (metastatic or non-metastatic). Cytoplasmic/nuclear staining was considered to indicate positive CacyBP expression and was scored on the following basis: 0 , no detectable staining; $1+,<25 \%$ positive cells; $2+, 25-49 \%$ positive cells; $3+, 50-74 \%$ positive cells; $4+,>75 \%$ positive cells. In general, cases demonstrating $3+$ and $4+$ staining were intensely stained, therefore, the level of intensity was not considered when calculating the score.

Clinicopathological assessment. The immunohistochemical results were compared against patient data and pathology

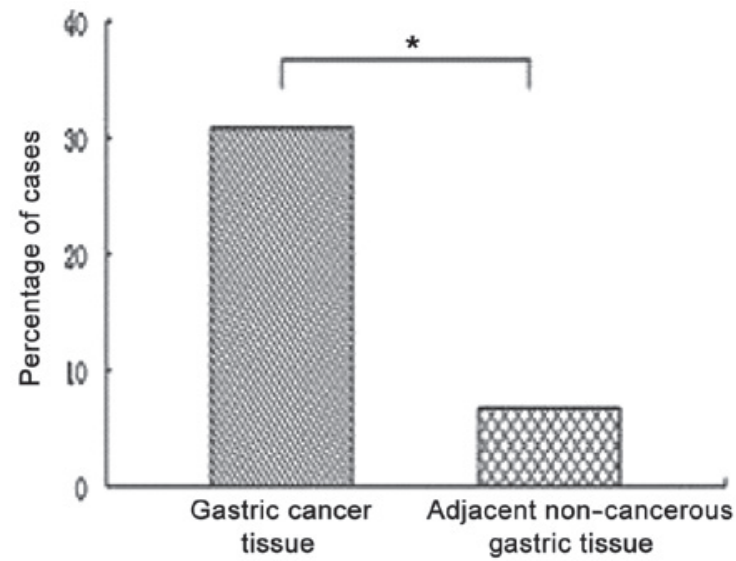

Figure 1. Expression of calcyclin binding protein/Siah-1-interacting protein (CacyBP/SIP) in gastric cancerous tissues and adjacent non-cancerous gastric tissues. The percentage of CacyBP/SIP immunoreactivity in these tissues is shown.

analysis of the tissue slices. The slices were assessed for gender, age, degree of tumor differentiation and TNM stage.

Western blot analysis. Tissue samples of gastric adenocarcinoma were lysed in $300 \mu \mathrm{l}$ freshly prepared extraction buffer [1\% SDS, $1 \mathrm{mmol} / 1 \mathrm{Na}_{3} \mathrm{VO}_{4}$ and $0.1 \mathrm{~mol} / 1$ Tris (pH 7.4)] and the proteins ( $40 \mu \mathrm{g} / \mathrm{lane})$ were resolved on $12 \%$ SDS-polyacrylamide gels, prior to being electrophoretically transferred to polyvinylidene difluoride membranes (EMD Millipore, Bedford, MA, USA) for 20-50 min at $20 \mathrm{~V}$. The membranes were incubated at $4^{\circ} \mathrm{C}$ overnight with one of the following primary monoclonal antibodies: CacyBP/SIP (dilution, 1:1,000) or mouse anti-canine $\beta$-actin (dilution, 1:2,000; cat. no. A2228; Sigma Aldrich, St. Louis, MO, USA). Subsequently, the membranes were incubated with monoclonal goat anti-mouse $\operatorname{IgG}$ secondary antibody (dilution, 1:2,000; cat. no. RPN5782; GE Healthcare Life Sciences, Piscataway, NJ, USA) and were detected using a SuperSignal West Pico Chemiluminescent Substrate kit (Pierce Biotechnology, Inc., Rockford, IL, USA). For each western blotting result, a minimum of three independent experiments were conducted; representative images are indicated in the results section.

Statistical analysis. Statistical analysis was performed using SPSS software (version 10.0; SPSS Inc., Chicago, IL, USA). The $\chi^{2}$ test and Fisher's exact test were used to determine the significance of the difference in frequency of CacyBP/SIP expression levels between non-metastatic and metastatic gastric cancer. $\mathrm{P}<0.05$ was considered to indicate a statistically significant difference.

\section{Results}

Expression levels of CacyBP/SIP. Among the 181 cases, the rates of CacyBP/SIP expression were 31\% (56/181) in cancerous and $7 \%(12 / 181)$ in adjacent non-cancerous gastric tissues, thus, the CacyBP/SIP expression rate was significantly higher in the cancerous gastric tissue samples $(\mathrm{P}<0.05$; Fig. 1). In the majority of cancer cells, CacyBP/SIP-positive staining was observed simultaneously in the cytoplasm and nuclei (Fig. 2A 

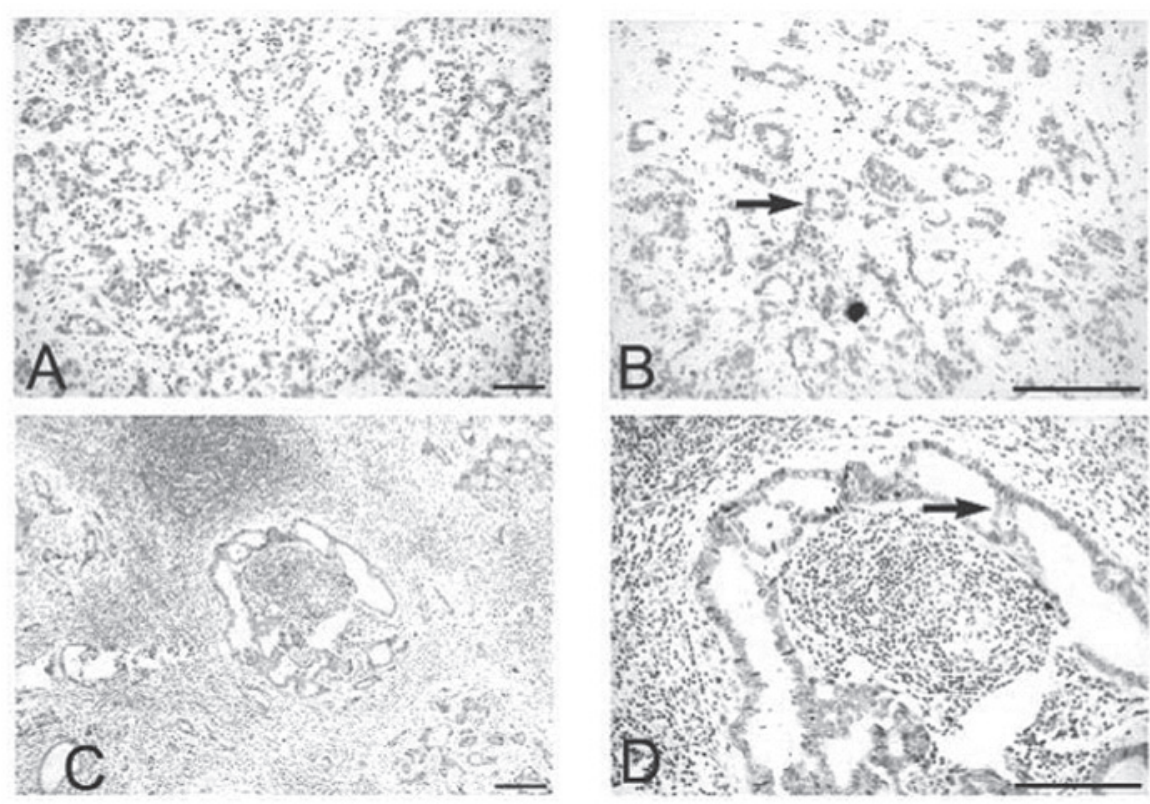

Figure 2. Expression levels of calcyclin binding protein/Siah-1-interacting protein (CacyBP/SIP) at primary and metastatic sites of patients with gastric cancer Strong CacyBP/SIP expression levels were identified at (A and B) a primary and (C and D) a lymph node metastatic site of a single patient. The CacyBP/SIP expression in these images was categorized as $3+, 4+, 3+$ and $4+$, respectively. Arrows indicate sites of diffuse staining in the cytoplasm and nucleus. Magnification of $\mathrm{A}$ and $\mathrm{C}$, x40; magnification of B and D, x100; scale bar, $50 \mu \mathrm{m}$.

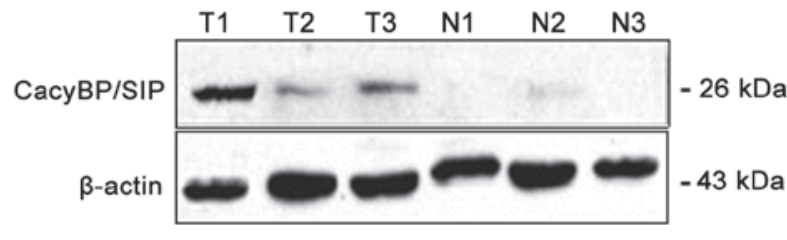

Figure 3. Western blot analysis of calcyclin binding protein/Siah-1-interacting protein expression levels in gastric cancer tissues $(\mathrm{T})$ and adjacent non-cancerous gastric tissues $(\mathrm{N})$.

and B). Additionally, CacyBP/SIP expression levels were examined by performing western blot analysis on the cancerous and adjacent non-cancerous gastric tissues taken from three patients. Western blotting demonstrated that CacyBP/SIP expression levels were higher in the three cancerous gastric tissue samples compared with the corresponding adjacent non-cancerous gastric tissue samples (Fig. 3).

CacyBP/SIP expression levels and clinicopathological features. The expression levels of CacyBP/SIP in the patient samples were compared against the clinicopathological findings of the patients (Table I). No significant association was identified between CacyBP/SIP expression and patient age $(\mathrm{P}=0.975)$, gender $(\mathrm{P}=0.185)$, degree of tumor differentiation $(\mathrm{P}=0.076)$ or TNM stage $(\mathrm{P}=0.979)$.

Expression levels of CacyBP/SIP in metastatic gastric cancer. To investigate whether an association exists between the expression levels of CacyBP/SIP and metastasis of gastric cancer, the expression levels of CacyBP/SIP were compared between primary tumor sites and the corresponding lymph node metastatic sites in 101 patients with metastatic gastric cancer. CacyBP/SIP expression was detected as cytoplasmic/nuclear staining of variously differentiated cancer tissues (Fig. 2C and D), consistent with our previous study (7). In the present study, CacyBP/SIP was expressed at primary sites in 31\% (31/101) of patients and at metastatic sites in $26 \%(26 / 101)$ of patients, thus, no significant difference was identified in the level of CacyBP/SIP expression between the primary and metastatic sites of the same metastatic gastric cancer patients $(\mathrm{P}=0.434$; Table II).

Subsequently, the expression levels of CacyBP/SIP were compared between the primary tumor sites of 80 patients with non-metastatic gastric cancer and the metastatic tumor sites of 101 patients with metastatic gastric cancer. As indicated in Table III, CacyBP/SIP was expressed in 34\% (27/80) of the non-metastatic gastric cancer patients and in 31\% (31/101) of the metastatic gastric cancer patients. No significant difference was identified in the frequency of CacyBP/SIP expression between the two groups of patients $(\mathrm{P}=0.662)$.

\section{Discussion}

Previous studies identified that overexpression of CacyBP/SIP inhibits the proliferation of gastric cancer cells, suppresses tumorigenesis in vitro and prolongs the survival of tumor-bearing nude mice $(14,18)$. Additionally, downregulation of CacyBP/SIP by RNA interference has the opposite effects. These findings indicate that CacyBP/SIP acts as a suppressor in gastric cancer, however, the expression of CacyBP/SIP in gastric cancerous tissue is unknown. In the present study, the expression levels of CacyBP/SIP were evaluated in clinical samples from gastric cancer patients. The results indicated that the level of CacyBP/SIP expression in gastric cancer tissues is significantly higher compared with the adjacent non-cancerous gastric tissues ( 31 vs. $7 \%$; $\mathrm{P}<0.05$ ); this was confirmed by western blot analysis. However, subsequent analysis of the clinicopathological features revealed that the expression levels of CacyBP/SIP were not associated with clinicopathological factors of gastric 
Table I. Association of CacyBP/SIP expression levels with clinicopathological features of gastric cancer $(n=181)$.

\begin{tabular}{|c|c|c|c|c|c|c|c|}
\hline \multirow[b]{2}{*}{ Category } & \multirow[b]{2}{*}{$\mathrm{n}(\%)$} & \multicolumn{5}{|c|}{ Level of CacyBP/SIP expression, $n$} & \multirow[b]{2}{*}{ P-value } \\
\hline & & 0 & $1+$ & $2+$ & $3+$ & $4+$ & \\
\hline \multicolumn{8}{|l|}{ Gender } \\
\hline Male & $109(60)$ & 70 & 17 & 17 & 3 & 2 & 0.185 \\
\hline Female & $72(40)$ & 53 & 11 & 6 & 2 & 1 & \\
\hline \multicolumn{8}{|l|}{ Age, years } \\
\hline$<50$ & $59(33)$ & 40 & 7 & 9 & 2 & 1 & 0.975 \\
\hline$\geq 50$ & $122(67)$ & 83 & 20 & 14 & 3 & 2 & \\
\hline \multicolumn{8}{|l|}{ Tumor differentiation } \\
\hline Well-differentiated & $50(28)$ & 38 & 5 & 5 & 1 & 1 & 0.076 \\
\hline Moderately differentiated & $39(22)$ & 21 & 10 & 6 & 1 & 1 & \\
\hline Poorly differentiated & $92(50)$ & 64 & 12 & 12 & 3 & 1 & \\
\hline \multicolumn{8}{|l|}{ TNM stage } \\
\hline $\mathrm{I}+\mathrm{II}$ & $84(46)$ & 57 & 12 & 10 & 3 & 2 & 0.979 \\
\hline III + IV & $97(54)$ & 66 & 15 & 13 & 2 & 1 & \\
\hline
\end{tabular}

CacyBP/SIP, calcyclin binding protein/Siah-1-interacting protein; TNM, tumor, node and metastasis. P-value represents positive staining compared with negative staining.

Table II. Comparison of CacyBP/SIP expression levels between primary and metastatic sites from the same gastric cancer patients.

\begin{tabular}{|c|c|c|c|c|c|c|c|}
\hline \multirow[b]{2}{*}{ Tumor site } & \multirow[b]{2}{*}{$\mathrm{n}$} & \multicolumn{5}{|c|}{$\begin{array}{l}\text { Level of CacyBP/SIP } \\
\text { expression, } n\end{array}$} & \multirow[b]{2}{*}{ P-value } \\
\hline & & 0 & $1+$ & $2+$ & $3+$ & $4+$ & \\
\hline Primary & 101 & 70 & 14 & 12 & 3 & 2 & 0.434 \\
\hline Metastatic & 101 & 75 & 12 & 9 & 2 & 3 & \\
\hline
\end{tabular}

P-value represents positive staining compared with negative staining. CacyBP/SIP, calcyclin binding protein/Siah-1-interacting protein.

cancer, such as age, gender, tumor size, degree of tumor differentiation or TNM stage. According to the results of the present study, CacyBP/SIP may not act as a tumor suppressor in gastric cancer as it is highly expressed in gastric cancer tissue. These conflicting results require additional studies to be performed to clarify the role of CacyBP/SIP in gastric cancer.

CacyBP/SIP expression was detected in only $31 \%$ of the gastric cancer samples using immunohistochemistry, however, it was detected in $100 \%$ of the samples using western blotting. This discrepancy may be due to differences in the sensitivity of the two assays or due to a statistical anomaly, as only three samples were assessed using western blot analysis. Furthermore, a significantly higher frequency of CacyBP/SIP expression was identified in the nuclei of cancerous gastric tissue; thus, further studies are required to explore the possible role of CacyBP/SIP in gastric cancer.
Table III. Comparison of CacyBP/SIP expression levels at primary tumor sites in patients with metastatic gastric cancer and patients with non-metastatic gastric cancer.

Level of CacyBP/SIP expression, $\mathrm{n}$

Type of

$\begin{array}{llllllll}\text { gastric cancer } & \mathrm{n} & 0 & 1+ & 2+ & 3+ & 4+ & \text { P-value }\end{array}$

\begin{tabular}{llllllll}
\hline Metastatic & 101 & 70 & 14 & 12 & 3 & 2 & 0.662
\end{tabular}

(lymph node)

$\begin{array}{lllllll}\text { Non-metastatic } & 80 & 53 & 13 & 11 & 2 & 1\end{array}$

P-value represents positive staining compared with negative staining. CacyBP/SIP, calcyclin binding protein/Siah-1-interacting protein.

Various studies have identified an association between CacyBP/SIP expression levels and different types of cancer metastasis. In the present study, the association between CacyBP/SIP expression levels and gastric cancer metastasis was investigated; however, no evidence of an association was detected. No significant difference was identified in the expression levels of CacyBP/SIP between primary and metastatic gastric cancer sites from the same patients. In addition, no significant difference was identified in the expression levels of CacyBP/SIP between non-metastatic and metastatic gastric cancer tissue from different patients.

Unlike breast and pancreatic cancer, in which CacyBP/SIP is involved in metastasis, CacyBP/SIP does not appear to be involved in the metastasis of gastric cancer. The progression of breast and pancreatic cancer are associated with 
CacyBP/SIP expression or overexpression, whereas the present study identified that overexpression of CacyBP/SIP results in the suppression of renal and gastric cancer cell growth. Thus, the present study proposes that CacyBP/SIP behaves differently in different types of cancer, and these differences should be the focus of future studies. It is possible that CacyBP/SIP has tumor-specific roles, similar to silent information regulator 1, nuclear factor- $\kappa \mathrm{B}$ and transforming growth factor- $\beta$ (19-21).

Although CacyBP/SIP is important in the tumorigenesis of cancer, the present study indicates that CacyBP/SIP may promote tumor suppression as opposed to being involved in the metastasis of gastric cancer. Furthermore, CacyBP/SIP expression levels do not appear to be associated with common clinicopathological features of gastric cancer. In conclusion, the results of the present study indicate that CacyBP/SIP plays a different role in gastric cancer than it does in breast or pancreatic cancer.

\section{Acknowledgements}

The present study was supported by the National Natural Science Foundation of China (grant no. 81072040).

\section{References}

1. Filipek A and Kuźnicki J: Molecular cloning and expression of a mouse brain cDNA encoding a novel protein target of calcyclin. J Neurochem 70: 1793-1798, 1998.

2. Matsuzawa SI and Reed JC: Siah-1, SIP, and Ebi collaborate in a novel pathway for beta-catenin degradation linked to p53 responses. Mol Cell 7: 915-926, 2001.

3. Yang YJ, Liu WM, Zhou JX, et al: Expression and hormonal regulation of calcyclin-binding protein (CacyBP) in the mouse uterus during early pregnancy. Life Sci 78: 753-760, 2006.

4. Au KW, Kou CY, Woo AY, et al: Calcyclin binding protein promotes DNA synthesis and differentiation in rat neonatal cardiomyocytes. J Cell Biochem 98: 555-566, 2006.

5. Herington JL, Bi J, Martin JD and Bany BM: Beta-catenin (CTNNB1) in the mouse uterus during decidualization and the potential role of two pathways in regulating its degradation. J Histochem Cytochem 55: 963-974, 2007.
6. Schneider G, Nieznanski K, Kilanczyk E, et al: CacyBP/SIP interacts with tubulin in neuroblastoma NB2a cells and induces formation of globular tubulin assemblies. Biochim Biophys Acta 1773: 1628-1636, 2007.

7. Filipek A, Schneider G, Mietelska A, Figiel I and Niewiadomska G: Age-dependent changes in neuronal distribution of CacyBP/SIP: comparison to tubulin and the tau protein. J Neural Transm 115: 257-264, 2008.

8. Kilanczyk E, Filipek S, Jastrzebska B and Filipek A: CacyBP/SIP binds ERK $1 / 2$ and affects transcriptional activity of Elk-1. Biochem Biophys Res Commun 380: 54-59, 2009.

9. Liang J, Luo G, Ning X, et al: Differential expression of calcium-related genes in gastric cancer cells transfected with cellular prion protein. Biochem Cell Biol 85: 375-383, 2007.

10. Shi Y, Hu W, Yin F, et al: Regulation of drug sensitivity of gastric cancer cells by human calcyclin-binding protein (CacyBP). Gastric Cancer 7: 160-166, 2004

11. Zhai H, Shi Y, Yu J, et al: Establishment and characterization of calcyclin binding protein (CacyBP) monoclonal antibody. Hybridoma (Larchmt) 25: 91-94, 2006.

12. Zhai H, Shi Y, Jin H, et al: Expression of calcyclin-binding protein/Siah-1 interacting protein in normal and malignant human tissues: an immunohistochemical survey. J Histochem Cytochem 56: 765-772, 2008.

13. Sun S, Ning X, Liu J, et al: Overexpressed CacyBP/SIP leads to the suppression of growth in renal cell carcinoma. Biochem Biophys Res Commun 356: 864-871, 2007.

14. Ning X, Sun S, Hong L, et al: Calcyclin-binding protein inhibits proliferation, tumorigenicity, and invasion of gastric cancer. Mol Cancer Res 5: 1254-1262, 2007.

15. Chen X, Han G, Zhai H, et al: Expression and clinical significance of CacyBP/SIP in pancreatic cancer. Pancreatology 8: 470-477, 2008.

16. Wang N, Ma Q, Wang Y, Ma G and Zhai H: CacyBP/SIP expression is involved in the clinical progression of breast cancer. World J Surg 34: 2545-2552, 2010.

17. Ajani JA, Bentrem DJ and Besh S, et al; National Comprehensive Cancer Network: Gastric cancer, version 2.2013: featured updates to the NCCN Guidelines. J Natl Compr Canc Netw 11: 531-546, 2013.

18. Ning X, Sun S, Zhang K, et al: S100A6 protein negatively regulates CacyBP/SIP-mediated inhibition of gastric cancer cell proliferation and tumorigenesis. PLoS One 7: e30185, 2012.

19. Deng CX: SIRT1, is it a tumor promoter or tumor suppressor? Int J Biol Sci 5: 147-152, 2009.

20. Perkins ND: NF-кB: tumor promoter or suppressor? Trends Cell Biol 14: 64-69, 2004.

21. Bachman KE and Park BH: Duel nature of TGF- $\beta$ signaling: tumor suppressor vs. tumor promoter. Curr Opin Oncol 17: 49-54, 2005. 